\title{
Durability of Polymer Powder Coatings
}

\author{
Vladimir Fedyaev ${ }^{1, *}$,Engel Galimov², Alexey Belyaev², and Liliya Sirotkina ${ }^{3}$ \\ ${ }^{1}$ Institute of Mechanics and Engineering of FIC KazanSC of RAS, 420111, Kazan, Russia \\ ${ }^{2}$ Kazan National Research Technical University named after A.N. Tupolev-KAI, 420111, Kazan, \\ Russia \\ ${ }^{3}$ Kazan State Energy University, 420066, Kazan, Russia
}

\begin{abstract}
The durability of polymer powder coatings is considered. The features of the impact on coatings during their operation of the environment, loads from the side of the substrate on the surface of its contact with the coatings, parameters that depend on the chemical composition and structure of the coating material are briefly analyzed. The thickness of the coating stands out as the main indicators of durability; a function that characterizes the continuity, strength, corrosion resistance, and its other properties; adhesive strength. It is proposed to use a generalized (integral) criterion for a comprehensive accounting of these indicators. An equation is written to assess the durability of the considered coatings, in a particular case, a formula is given for calculating their permissible operating time.
\end{abstract}

\section{Introduction}

The beginning of the industrial use of polymer powder paints dates back to the 50s of the last century. At present, they are successfully used at enterprises of mechanical engineering, the fuel and energy complex, chemistry, agriculture and communal services, and many other industries. There are two main types of polymer powder materials: thermosetting and thermoplastic powder compositions (PPC). Coatings based on thermosetting PPC are highly resistant to acids, alkalis, and other chemically aggressive substances; increased mechanical properties: elasticity, impact strength, as well as adhesive strength indicators. Thermoplastic powder compositions are convenient for storage, are characterized by a short coating formation cycle, better sanitary and hygienic working conditions, and less fire hazard. In addition, they are resistant to wear, have high electrical insulation and decorative performance $[1,2]$.

Undoubtedly, a very important characteristic of the operational properties of polymer powder coatings is their durability. In accordance with the regulatory documents, the durability of coatings is determined by lightfastness, resistance to aggressive liquid media, atmospheric factors (pressure, temperature, humidity, etc.) [3-6]. With regard to equipment, technological installations, technical systems of the oil industry, as the main factors affecting the durability of the considered coatings, an aggressive environment, temperature and mechanical loads are distinguished [7]. Ultimately, durability is the lifetime of coatings during which they are able to perform the required functions.

\footnotetext{
*Corresponding author: vlfed2020@gmail.com
} 
For the sake of certainty, let us dwell on the case when polymer powder coatings are operated in air, respectively, a gaseous medium containing oxidizing agents acts on their outer surface: oxygen, ozone; chemically active substances (pollution); water in the form of drops, steam; mechanical (abrasive) impurities. Further, the coatings can be affected by light, ultraviolet, infrared (radiation) radiation; temperature loads; loads in the form of pressure, concentrated forces and moments, impacts, etc. In general, four main factors act on the outside of the coating - air, radiation, temperature, mechanical stress.

\section{Main part}

In order to further, when assessing the durability of coatings, it would be possible to use the methods of mathematical modeling, let us denote through $\tau_{\mathrm{k}}$ the time characterizing the durability, through $Q_{\mathrm{a}}, Q_{\mathrm{r}}, Q_{t}, Q_{\mathrm{m}}$ - the intensity of the impact on the outer surface of the coating of air, radiation, temperature and mechanical loads. Moreover, each of these indicators is integral, reflecting, as a rule, the effect of several factors. For example, $Q_{\text {a }}$ depends on the concentration of oxidants, chemically active substances, humidity, mechanical impurities in the air; $Q_{\mathrm{t}}$ - from the actual ambient temperature, temperature differences, the magnitude of heat fluxes. If it is required to assess in more detail the influence of individual factors, then the values of $Q_{\mathrm{a}}, Q_{\mathrm{r}}, Q_{\mathrm{t}}, Q_{\mathrm{m}}$ can be interpreted as vectors, the components of which estimate the intensity of the impact on the outer surface of the coating of these factors.

At the same time, from the side of the substrate, on the surface of its contact with the coating layer, which we will call the inner surface, factors of a chemical and physical nature can act, let us denote their intensity by qc and $q_{\mathrm{f}}$, respectively, as well as temperature and mechanical factors characterized by the intensity $q_{\mathrm{t}}$ and $q_{\mathrm{m}}$, respectively.

Finally, a set of so-called internal factors should be distinguished, due to the chemical composition of the coating, the presence of weak bonds, microcracks and pores, internal stresses, etc. The degree of influence of these factors on the performance of the material will be denoted by $m_{\mathrm{c}}, m_{s}, m_{\mathrm{p}}, m_{\mathrm{e}}$, etc.

Obviously, during the operation of coatings, almost all of these factors depend to one degree or another on the time $\tau$ :

$$
\begin{gathered}
Q_{\mathrm{a}}=Q_{\mathrm{a}}(\tau), Q_{\mathrm{r}}=Q_{\mathrm{r}}(\tau), Q_{\mathrm{t}}=Q_{\mathrm{t}}(\tau), Q_{\mathrm{m}}=Q_{\mathrm{m}}(\tau) ; \\
q_{\mathrm{c}}=q_{\mathrm{c}}(\tau), q_{\mathrm{f}}=q_{\mathrm{f}}(\tau), q_{\mathrm{t}}=q_{\mathrm{t}}(\tau), q_{\mathrm{m}}=q_{\mathrm{m}}(\tau) ; \\
m_{\mathrm{c}}=m_{\mathrm{c}}(\tau), m_{\mathrm{s}}=m_{\mathrm{s}}(\tau), m_{\mathrm{p}}=m_{\mathrm{p}}(\tau), m_{\mathrm{e}}=m_{\mathrm{e}}(\tau) .
\end{gathered}
$$

To simplify the subsequent entries, the intensity of the combination of factors acting on the outer surface of the coating will be denoted by $Q_{0}=Q_{0}(\tau)$; on the inner surface (the contact surface of the coating layer and the base) $-q_{\mathrm{b}}=q_{\mathrm{b}}(\tau)$; the intensity of the factors characterizing the properties of the coating material $-m_{\mathrm{i}}=m_{\mathrm{i}}(\tau)$. Moreover, $Q_{0}, q_{\mathrm{b}}, m_{\mathrm{i}}$ are essentially functionals:

$$
Q_{0}=Q_{0}\left(Q_{\mathrm{a}}, Q_{\mathrm{r}}, Q_{\mathrm{t}}, Q_{\mathrm{m}}\right), q_{\mathrm{b}}=q_{\mathrm{b}}\left(q_{\mathrm{c}}, q_{\mathrm{f}}, q_{\mathrm{t}}, q_{\mathrm{m}}\right), m_{\mathrm{i}}=m_{\mathrm{i}}\left(m_{\mathrm{c}}, m_{\mathrm{s}}, m_{\mathrm{p}}, m_{\mathrm{e}}, \ldots\right) .
$$

In the presence of factors, the action of loads $Q_{0}, q_{\mathrm{b}}$ on the coating, mechanical and physicochemical processes occur in the coating material, it can break down, peel off from the substrate, etc. [6]. In fact, aging of the coating material occurs, which has been studied and described in sufficient detail in the scientific and technical literature [8-10]. When using 
the data presented in it, the following circumstances must be taken into account: the relatively small thickness of the coating layer, the impact on it of both loads from the outer surface and those acting on the inner surface of the contact of the coating layer with the substrate. Accordingly, the adhesion of the coating material to the substrate should be considered, and the adhesion strength should be evaluated [11-13].

In this situation, it is advisable to choose as indicators characterizing the durability of polymer powder coatings, the thickness of the coating $H_{\mathrm{c}}$; functional $G_{\mathrm{c}}$, describing the continuity, strength, corrosion resistance, other performance properties of the coating material, as well as the adhesion strength $F_{\mathrm{a}}$. These indicators, like all the previous ones, also depend on the operating time $\tau$ :

$$
H_{\mathrm{c}}=H_{\mathrm{c}}(\tau), G_{\mathrm{c}}=G_{\mathrm{c}}(\tau), F_{\mathrm{a}}=F_{\mathrm{a}}(\tau)
$$

Obviously, the time for them to reach the critical dependences $H_{\mathrm{ck}}, G_{\mathrm{ck}}, F_{\mathrm{ak}}$, due to the corresponding requirements for the functionality of the considered coatings, is different. But if each of them is important, it is naturally desirable to take all of them into account when assessing the durability of coatings $\tau_{\mathrm{k}}$. One of the possible ways to solve this problem is to construct a generalized (integral) criterion

$$
K=K(\tau)=F\left(H_{\mathrm{c}}(\tau), G_{\mathrm{c}}(\tau), F_{\mathrm{a}}(\tau)\right)
$$

In the simplest case, the functional $\mathrm{K}$ can be represented as:

$$
K=K(\tau)=\alpha H_{\mathrm{c}}(\tau)+\beta G_{\mathrm{c}}(\tau)+\gamma F_{\mathrm{a}}(\tau)
$$

where $H_{\mathrm{c}}, G_{\mathrm{c}}, F_{\mathrm{a}}$ - normalized functions; $\alpha, \beta, \gamma$-weight coefficients characterizing the significance of the corresponding indicator $(0 \leq \alpha \leq 1,0 \leq \beta \leq 1,0 \leq \gamma \leq 1 ; \alpha+\beta+\gamma=1)$. In particular, if $\alpha=\beta=0, \gamma=1$, then the adhesion strength of the coating is dominant. If, for example, $\alpha=0.75, \beta=0.25, \gamma=0$, the focus is on the wear of the coating material during its operation. In our opinion, the most effective way to establish the values of these coefficients is an expert one.

Since, as noted above, it is required that the values of the indicators $H_{\mathrm{c}}, G_{\mathrm{c}}, F_{\mathrm{a}}$ are not less than the critical $H_{\mathrm{ck}}, G_{\mathrm{ck}}, F_{\mathrm{ak}}$, the equation for finding the value $\mathrm{K}$ follows from the expression for the criterion $\tau_{\mathrm{k}}$ :

$$
K\left(\tau_{\mathrm{k}}\right)=K_{\mathrm{k}}
$$

Here $K_{\mathrm{k}}=\alpha H_{\mathrm{ck}}+\beta G_{\mathrm{ck}}+\gamma F_{\mathrm{a}}-$ a parameter characterizing the limitations imposed on individual indicators of the durability of coatings and the significance of each of them.

Practice shows that by the end of the service life of the coatings, the functions $H_{\mathrm{c}}(\tau)$, $G_{\mathrm{c}}(\tau), F_{\mathrm{a}}(\tau)$ are close to linear, respectively,

$$
H_{\mathrm{c}}(\tau)=a_{0}+a_{1} \tau, G_{\mathrm{c}}(\tau)=b_{0}+b_{1} \tau, F_{\mathrm{a}}(\tau)=c_{0}+c_{1} \tau,
$$

where the coefficients are $a_{0}, a_{1} ; b_{0}, b_{1} ; c_{0}, c_{1}$ are determined as a result of analysis of literature data, experiments, theoretical research.

From this it is clear that the durability of polymer coatings can be calculated by the formula:

$$
\tau_{\mathrm{k}}=\left(K_{\mathrm{k}}-D_{0}\right) / D_{1}
$$

where $D_{0}=\alpha a_{0}+\beta_{\mathrm{b} 0}+\gamma_{\mathrm{c} 0}, D_{1}=\alpha a_{1}+\beta_{\mathrm{b} 1}+\gamma_{\mathrm{cl}}$. 


\section{Conclusions}

Taking into account the specifics of obtaining polymer powder coatings, their properties, operating conditions, an approach is proposed, and the main provisions for assessing the durability $\tau_{\mathrm{k}}$ of these coatings are developed. Provided that the thickness of the coating layer and the strength of adhesion are chosen as the main indicators of durability, an equation for finding $\tau_{\mathrm{k}}$ is written. In the particular case of a linear dependence of these indicators on the operating time, setting their critical values, a formula for calculating the value $\tau_{\mathrm{k}}$ is determined.

\section{References}

1. A.D. Yakovlev, Powder paints (Chemistry, Leningrad, 1987)

2. E.R. Galimov, E.V. Zverev, E.E. Tukbaev et al., Polymer powder coatings for special purposes (Offset Service, Kazan, 2021)

3. L.A. Sukharev, Durability of polymer coatings (Chemistry, Moscow, 1984)

4. A.V. Sukhodolya, N.V. Odinokova, Modern approaches to predicting the durability of paint and varnish coatings of construction and road machines, Construction and road machines, v. 5, pp. 26-29 (2014)

5. V.T. Erofeev, I.V. Smirnov, P.V. Voronov, V.V. Afonin, E.N. Kablov, O.V. Startsev, V.O. Startsev, I.M. Medvedev, Studies of the resistance of polymer coatings under the influence of climatic factors of the Black Sea coast, Fundamental research, v. 11, pp. 911-924 (2016)

6. E.R. Galimov, E.E. Tukbaev, V.L. Fedyaev et al., Highly efficient technologies and equipment for obtaining polymer powder coatings (Publishing house of the Academy of Sciences of the Republic of Tatarstan, Kazan, 2016)

7. V.N. Protasov, Polymer coatings in the oil industry (Nedra, Moscow, 1985)

8. N.N. Pavlov, Aging of plastics in natural and artificial conditions (Chemistry, Moscow, 1982)

9. N.M. Emanuel, A.L. Buchachenko, Chemical physics of molecular destruction and stabilization of polymers (Nauka, Moscow, 1988)

10. M.T. Bryk, Destruction of filled polymers (Chemistry, Moscow, 1989)

11. A.D. Simon, Adhesion of films and coatings (Chemistry, Moscow, 1977)

12. A.A. Berlin, V.E. Basin, Basics of polymer adhesion (Chemistry, Moscow, 2003)

13. V.A. Bely, N.I. Egorenkov, Yu.M. Pleskachevsky, Adhesion of polymers to metals (Science and technology, Minsk, 2007) 